\title{
Universal Rolling Deformation of Asymmetric Unequal-leg Angles
}

\author{
Yukio TAKASHIMA, ${ }^{1) *}$ Naoki NAKATA ${ }^{1)}$ and Jun YANAGIMOTO ${ }^{21}$ \\ 1) Steel Research Laboratory, JFE Steel Corporation, Kawasakidori 1, Mizushima, Kurashiki, Okayama, $712-8511$ Japan. \\ 2) Institute of Industrial Science, The University of Tokyo, Komaba 4-6-1, Meguro, Tokyo, 153-8505 Japan.
}

(Received on March 2, 2014; accepted on June 11, 2014)

\begin{abstract}
Universal rolling of asymmetric unequal-leg angles having an L-section was studied by a model rolling experiment and finite element (FE) analysis. In the experiment, flange spread displayed a linear relationship against the reduction balance, which was defined as the difference of the flange and web thickness strains. Similar linear behaviors of web height, flange depth and bulge height against the reduction balance were also observed. Rolling deformation was also investigated in detail by FE simulation. Although the trends of deformation in the simulation were similar to the experimental results, two significant differences were noted: The inclination of the regression lines of deformation (flange spread parameter) was slightly different, and the change of flange depth was not linear in the high reduction balance region. Furthermore, unexpected web spread was observed in the experiment and FE analysis.

FE simulation of universal rolling of channels (U-section), in which the stock had the same half-section as the section of the unequal-leg angle (L-section), was carried out to investigate the similarities in the deformation behaviors of these two products. From a comparison of the upstream section in the two rolling simulations, unstable stock rotation at the entry of the rolls was discovered in unequal-leg angle rolling. The rotation angle was influenced by the reduction balance, and this rotation was considered to be the cause of the distinctive deformation observed in unequal-leg angle rolling. Finally, the importance of restricting rotation was examined with the aim of improving the stability of rolling deformation.
\end{abstract}

KEY WORDS: section rolling; model experiment; finite element analysis; spread; bulge.

\section{Introduction}

Universal rolling is widely applied in the production of sections such as H-beams, channels, rails, T-bars and similar products. Deformation properties in universal rolling have been studied in detail for H-beams, ${ }^{1,2)} \mathrm{T}^{- \text {bars }^{3)}}$ and channels. ${ }^{4)}$ As a common feature of these products, their cross sections are characterized by mirror symmetry. Specifically, the H-beam section has vertical and horizontal axes of symmetry, the section of a T-bar in universal rolling has a horizontal axis of symmetry and is similar to the left or right half of an H-beam, and a channel section has a vertical axis of symmetry and a cross section (U-section) similar to the upper or lower half of an H-beam.

The type of section symmetry is an important factor in universal rolling deformation. Rolled H-beams are usually straight upon delivery while T-bars often have side camber and channels mostly turns upward upon delivery. Section symmetry also influences rolling deformation in the cross section. For example, the flange spread behavior of a T-bar having a half-section of an H-beam is similar to that of the original H-beam. ${ }^{3)}$ In universal rolling of channels, it has been reported that overfill at the outside corners often occurs, as if two new flanges are created. ${ }^{4,5}$

Although the properties of universal rolling deformation

* Corresponding author: E-mail: y-takashima@jfe-steel.co.jp DOI: http://dx.doi.org/10.2355/isijinternational.54.2357 of products with symmetric cross sections have been studied extensively, only a few studies have examined asymmetric universal rolling. ${ }^{6-8)}$

To study deformation behavior in asymmetric universal rolling, an unequal-leg angle was selected for this research. An unequal-leg angle has an L-section, which is similar to a quarter section of an H-beam and the half-section of a channel and T-bar. Unequal-leg angles are commonly produced by groove rolling with a 2-high mill, ${ }^{9-11)}$ and the products are generally used in ships as stiffeners. ${ }^{12)}$ Because groove rolling requires a different roll pass design for each cross section, the size variety of rolled unequal-leg angles is limited. If universal rolling could be applied to the production of unequal-leg angles, it would be possible to products having different thicknesses with the same roll pairs, and the size variety could be expanded without increasing roll costs.

In this research, the basic deformation behavior of unequal-leg angles in universal rolling was investigated. A laboratory rolling experiment and finite element (FE) analysis were carried out under several conditions of web and flange thickness reduction. The deformation of flange width, web height, flange depth and bulge height was studied in detail.

In addition, FE simulation of rolling of a channel having the same half-section as the unequal-leg angle was executed. Based on a comparison of the rolling deformation of the unequal-leg angle and the channel, the characteristics of 
asymmetric universal rolling and the influence of symmetry on rolling deformation were discussed.

\section{Laboratory Rolling Experiment}

\subsection{Experimental Setup}

Figure 1 shows a schematic diagram of universal rolling of an unequal-leg angle. Although some results of angle rolling experiments with multi-roll mills have been reported, ${ }^{6-8)}$ the roll geometries and arrangements were different from those in H-beam universal rolling. In this research, the same horizontal and vertical rolls as those used in H-beam rolling were selected. Because the roll configuration and arrangement are the same, the common use of rolls between different products is possible.

Pure lead was used as the model material in all rolling experiments. It has been reported that the deformation behavior of pure lead is similar to that of hot carbon steel at elevated temperatures. ${ }^{13)}$ Because these experiments were carried out at room temperature, the effect of the temperature distribution of the workpiece on rolling deformation was eliminated.

A constant reduced-scale of one-fifth was assumed in the experiments. The reduced-scale was applied to both workpiece and rolls in order to maintain the geometric similarity of rolling deformation. ${ }^{13)}$ The dimensions of the rolls and workpiece cross section are shown in Table 1 and Fig. 2, respectively. The horizontal rolls of the mill were driven and

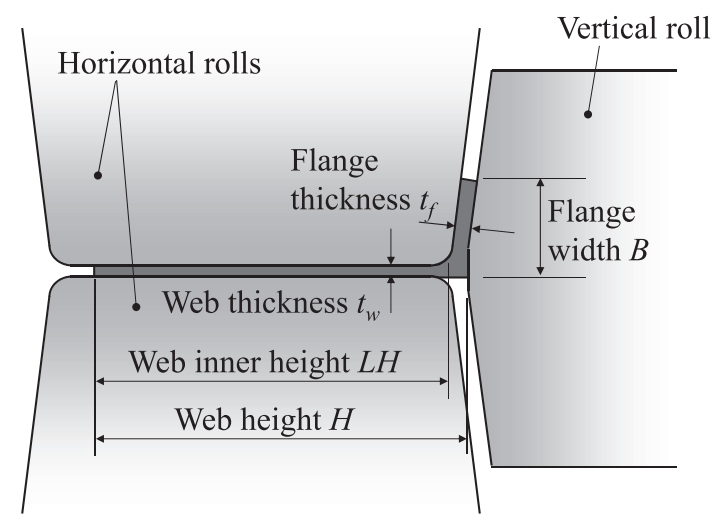

Fig. 1. Universal rolling of unequal-leg angle.

Table 1. Experimental conditions of laboratory universal mill.

\begin{tabular}{llc}
\hline $\begin{array}{l}\text { Roll diameter } \\
(\mathrm{mm})\end{array}$ & $\begin{array}{l}\text { Horizontal roll } \\
\text { Vertical roll }\end{array}$ & 180 \\
\hline Horizontal roll rotation speed & $7 \mathrm{rpm}$ \\
\hline Flange inclination angle & $6^{\circ}$ \\
\hline
\end{tabular}

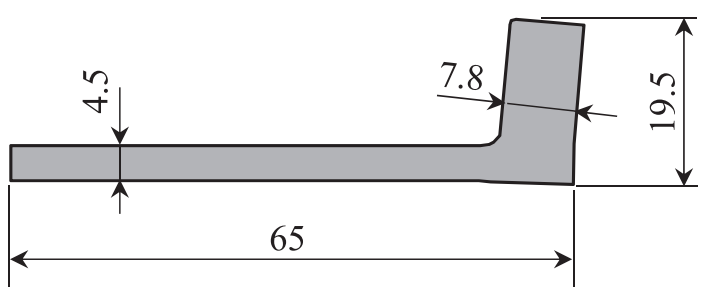

Fig. 2. Inlet section for rolling experiment. the vertical rolls were undriven. The workpiece length was set at $300 \mathrm{~mm}$. The geometry in this experiment corresponds closely to that of an intermediate pass when rolling an unequal-leg angle of $300 \times 90$, that is, an angle with a web height of $300 \mathrm{~mm}$ and flange width of $90 \mathrm{~mm}$.

The specimen was expected to turn up and curve sideways upon delivery, resulting in upward curling and side camber. Tables, web guides and vertical side plates were applied at both the entry and exit sides to enable delivery of straight rolled angles, as shown in Fig. 3. Although a smaller gap between the guides and the specimen increases the straightness of the rolled angle, an excessively small gap causes scratches on the surface of the workpiece. The gap was set at approximately $1 \mathrm{~mm}$ in the experiment.

\subsection{Modeling of Universal Rolling Deformation}

In research on H-beam universal rolling, the spread of flange width $B$ has been studied in detail, ${ }^{1,2)}$ and it has been shown that the difference between the flange thickness reduction $r_{f}$ and web thickness reduction $r_{w}$ strongly influences flange spread. That is, when $r_{f}$ is larger than $r_{w}$, flange elongation also becomes larger than that of the web. However, since the web and flange are connected, their elongations must be the same. This restriction on elongation results in larger flange spread under such rolling conditions. Conversely, when $r_{w}$ is larger, tensile force acts on the flange, and this tension reduces flange spread. This is the basic mechanism of the influence of rolling conditions on flange spread behavior in universal rolling.

Flange spread behavior in universal rolling is expressed by the following equation: ${ }^{1,2)}$

$$
\ln \left(B_{1} / B_{0}\right)=a \cdot\left(\lambda_{f}-\lambda_{w}\right)+b
$$

where $\lambda_{w}$ and $\lambda_{f}$ are the thickness strains of the web and flange, and are calculated using the following equations:

$$
\begin{gathered}
\lambda_{w}=\ln \left(t w_{0} / t w_{1}\right) \\
\lambda_{f}=\ln \left(t f_{0} / t f_{1}\right)
\end{gathered}
$$

where $t w$ and $t f$ are the thicknesses of the web and flange. The suffixes 0 and 1 in Eqs. (1) to (3) mean before and after rolling, respectively. In Eq. (1), $a$ and $b$ are constants depending on the dimensions of the product and the mill geometry. The difference between the flange and web thickness strain $\left(\lambda_{f}-\lambda_{w}\right)$ is considered to be the representative parameter of the correlation between the web and flange and

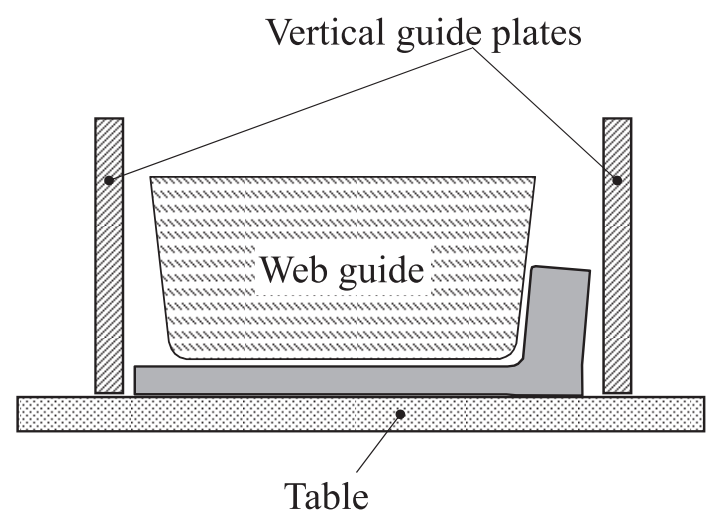

Fig. 3. Guide and table for rolling experiment. 
is called "reduction balance" in this paper.

Similar deformation properties have also been reported in universal rolling of T-bars ${ }^{3)}$ and channels. ${ }^{4)}$ Therefore, a similar influence was expected in universal rolling of unequal-leg angles.

\subsection{Experimental Conditions}

In order to examine the effect of rolling conditions on rolling deformation, several combinations of web thickness reduction $r_{w}$ and flange thickness reduction $r_{f}$ were tested. In universal mills, the horizontal and vertical roll gaps can be set individually. A target $r_{w}$ of $15 \%$ and a target range of $r_{f}$ between $12 \%$ and $24 \%$ were selected. As a result, the target range of the thickness reduction difference $\left(r_{f}-r_{w}\right)$ was $-3 \%$ to $9 \%$.

In the case of T-bar universal rolling, the tip of the web is a free surface, and web spread as been observed. ${ }^{3)}$ A similar web spread deformation was expected in rolling of unequal-leg angles. Therefore, in addition to the flange width, web height was also measured before and after the rolling experiment.

The flange of an unequal-leg angle is located on only one side of the web. A unique rolling deformation creating a bulge at the outside corners was observed in channel universal rolling. ${ }^{4,5)}$ As a similar bulging deformation, like that shown in Fig. 4, was also expected in unequal-leg angle rolling, the flange depth $d$ and bulge height $h$ were measured before and after rolling. The relationships between the reduction balance and changes in these dimensions were then investigated to validate the possibility of modeling as shown in Eq. (1).

\subsection{Experimental Results}

The rolling experiment was completed without any prob-

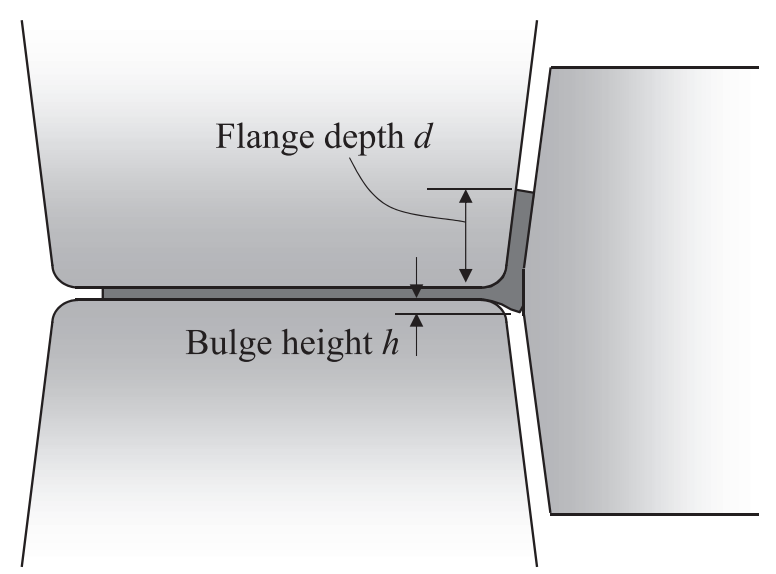

Fig. 4. Flange depth and bulge at outside corner. lems. Seven specimens were rolled under different rolling conditions. Figure 5 shows a photograph of a rolled pure lead specimen. Although all the rolled angles displayed upward curling and flange side camber at the top end, the web guide and vertical guide plates enabled delivery of the angles in a straight condition to the tail end.

The dimensions of the unequal-leg angles before and after rolling were measured at the center of length, and these measurements were used in calculating the actual thickness reductions, strains and spread deformation parameters. Figure 6 shows the actual web and flange thickness reductions. The range of $r_{w}$ was $14.9 \%$ to $16.6 \%$, while $r_{f}$ was 11.5 to $24.9 \%$. These reductions covered a sufficient range of $\left(r_{f}-r_{w}\right)$ corresponding to the planned experimental condition. The flange and web spread parameters, flange depth and bulge height were also evaluated using these measurements.

Figure 7 shows three rolled sections of pure lead specimens at the center of length. As can be seen in this figure, bulging deformation occurred, and the bulge height became larger with higher flange thickness reduction.

Figure 8 shows the change of the flange spread parameter $\ln \left(B_{1} / B_{0}\right)$ and the web inner height spread parameter $\ln \left(L H_{1} /\right.$ $\left.L H_{0}\right)$ against the reduction balance. Linear relationships can be seen between the two parameters and the reduction balance. However, $L H$ increased as the reduction balance increased. Based on the mechanism of universal rolling deformation, a higher reduction balance should decrease $L H$, as observed in T-bar universal rolling. ${ }^{3)}$ The cause of this unexpected tendency in the web inner height in angle rolling is discussed in Chapter 4.

The changes of the flange depth $\Delta d$ and bulge height $\Delta h$

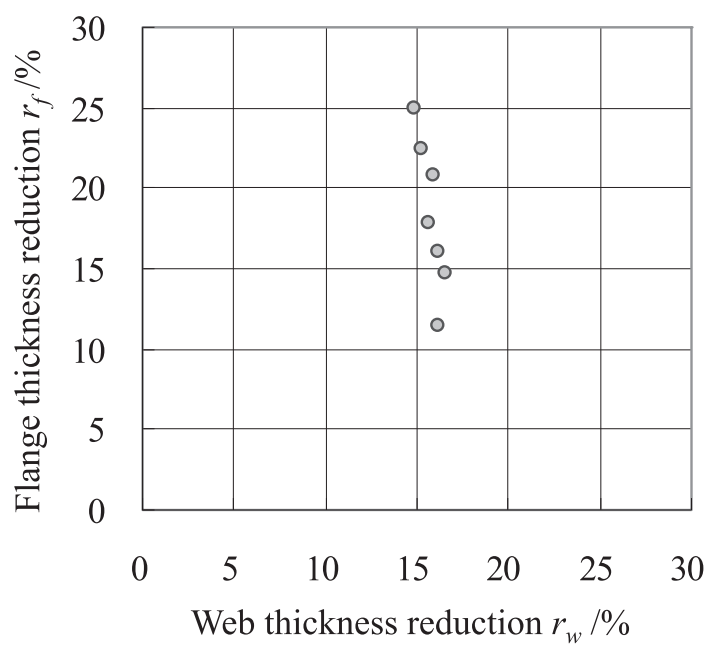

Fig. 6. Actual thickness reductions in experiment.

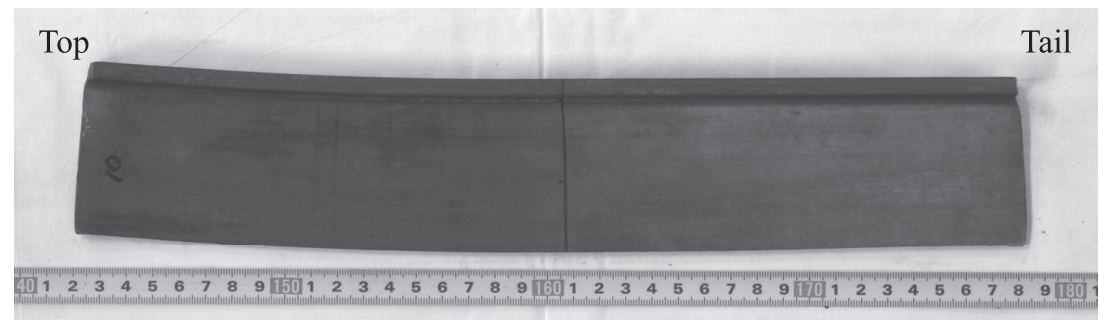

Fig. 5. Example of rolled pure lead specimen $\left(r_{w}=15.6 \%, r_{f}=17.8 \%\right)$. 


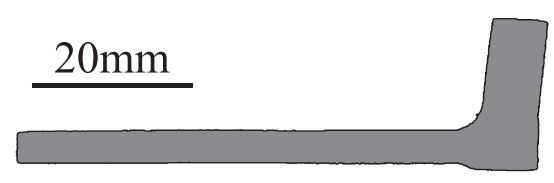

(a) $r_{w}=16.2 \%, r_{f}=11.5 \%$

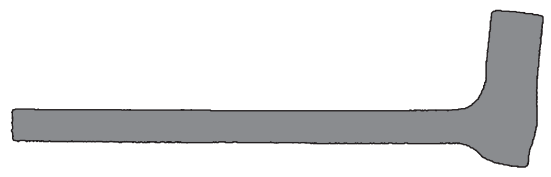

(b) $r_{w}=15.6 \%, r_{f}=17.8 \%$

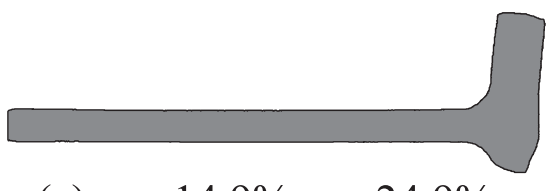

(c) $r_{w}=14.9 \%, r_{f}=24.9 \%$

Fig. 7. Sections after rolling in experiment.

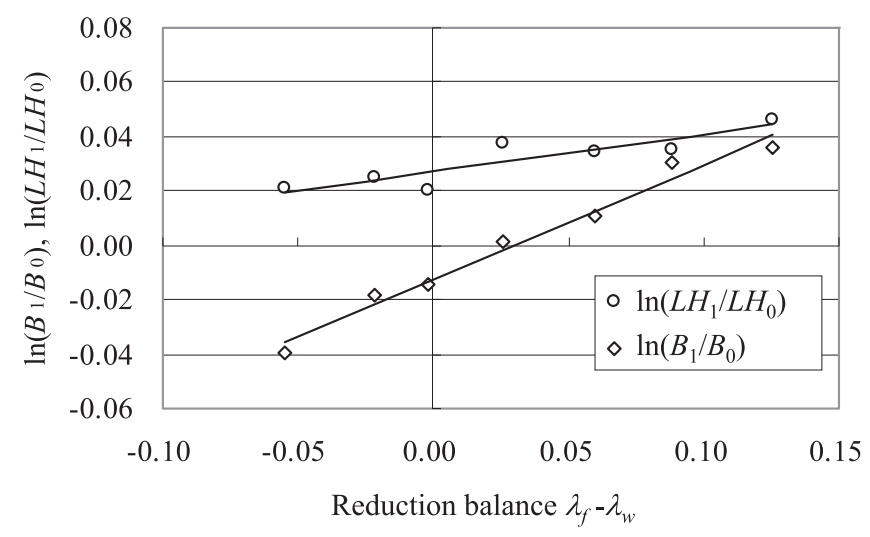

Fig. 8. Influence of reduction balance on flange and web spread (experiment).

against the reduction balance are presented in Fig. 9. The flange depth $\Delta d$ decreased in universal rolling while the bulge height $\Delta h$ increased. These values changed in a linear manner against the reduction balance.

From the results of the experiment, it can be understood that the influences of the reduction balance on $\ln \left(B_{1} / B_{0}\right)$, $\ln \left(L H_{1} / L H_{0}\right), \Delta d$ and $\Delta h$ can be expressed by the right side of Eq. (1).

\section{Finite Element Simulation of Angle Universal Roll- ing}

\subsection{Numerical Conditions}

In order to investigate deformation behavior in universal rolling of unequal-leg angles in detail, a three-dimensional numerical simulation was conducted. A dynamic explicit FE code, ABAQUS Explicit Ver.6.12, was used for the numerical analysis. Good agreement between the deformation in simulated rolling and that in the pure lead experiment was confirmed in our previous research. ${ }^{4)}$ Solid brick reduced integration elements (C3D8R) were used for the modeled angle, and the element numbers in the cross-section and length were 1228 and 376, respectively. Therefore, the element number of the workpiece was approximately 460000 .

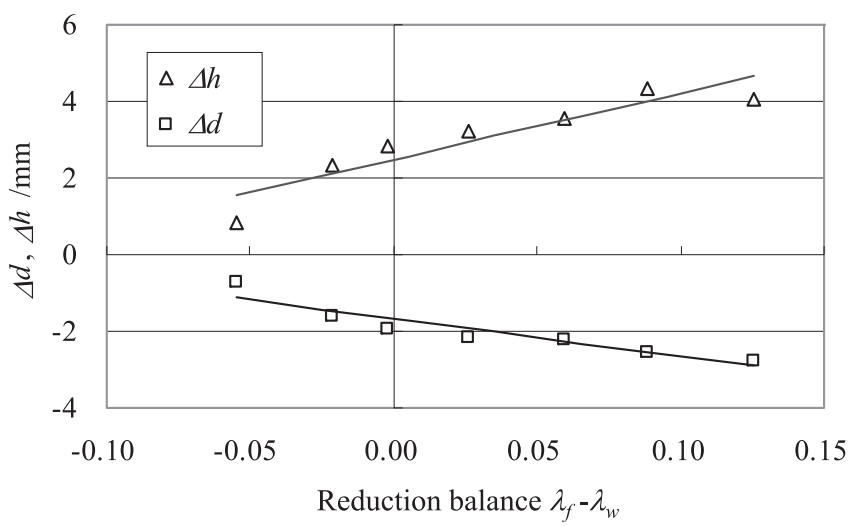

Fig. 9. Influence of reduction balance on flange depth and bulge height (experiment).

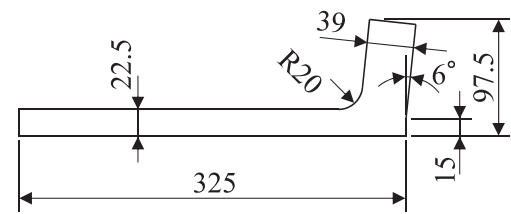

Fig. 10. Inlet section for FE analysis.

Table 2. Numerical conditions of FE simulation.

\begin{tabular}{lcc}
\hline \multicolumn{2}{c}{ Product size of unequal-leg angle } & $300 \times 90$ \\
\hline & Web height; $H$ & 325 \\
Inlet stock size (mm) & Flange width; $B$ & 97.5 \\
& Web thickness; $t w$ & 22.5 \\
& Flange thickness; $t f$ & 39 \\
\hline \multirow{2}{*}{ Roll diameter (mm) } & Horizontal roll & 900 \\
& Vertical roll & 700 \\
\hline Horizontal roll rotation speed & $40 \mathrm{rpm}$ \\
\hline Flange inclination angle & Web; $r_{w}$ & $6^{\circ}$ \\
\hline Target thickness & Flange; $r_{f}$ & $r_{w}=15 \%$ \\
reduction & Stock - roll & $r_{f}=12,15,18,21,24 \%$ \\
\hline Friction coefficient & Stock - table \& guide & 0.4 \\
\hline Young's modulus & & 0.0 \\
\hline Poisson ratio & & 0.3 \\
\hline
\end{tabular}

The rolls were assumed to be analytical rigid surfaces. In all cases, a non-steady-state model was used. The horizontal rolls were driven, and their rotation speed was set at $40 \mathrm{rpm}$. The vertical rolls were defined as undriven free rotation parts. The value of mass scaling was set to less than 50 in the simulations.

Real rolling geometries corresponding to the model rolling experiment in Chapter 2 were used in the numerical simulation. The dimensions of the one-fifth scale model in the experiment were multiplied by 5 times for conversion to the actual rolling geometry. Figure $\mathbf{1 0}$ shows the section geometry before rolling, and the numerical conditions are listed in Table 2. In the series of FE analyses, web thickness reduction was set at $15 \%$, and five flange reductions of $12 \%$, $15 \%, 18 \%, 21 \%$ and $24 \%$ were simulated. The web guides 
and tables in Fig. 3 were also constructed in the numerical model to avoid extreme upward curling and side camber in the simulation.

A flow stress curve for hot carbon steel ${ }^{14)}$ was used to create flow stress data for the analysis.

$$
\sigma_{f}=113.2 \cdot \varepsilon^{0.21} \cdot \dot{\varepsilon}^{0.13}
$$

where $\sigma_{f}$ is flow stress in $\mathrm{MPa}, \varepsilon$ is plastic strain and $\dot{\varepsilon}$ is the plastic strain rate. A constant rolling temperature of $1000^{\circ} \mathrm{C}$ and a carbon content of $0.15 \%$ were assumed in the stock. The flow stress calculated with strain of 0.002 in Eq. (4) was used as the initial flow stress for initiation of plastic deformation.

\subsection{Results of FE Analysis}

The FE simulations of the five conditions were executed successfully. Figure 11 illustrates a simulated rolling deformation with the target $r_{f}$ of $18 \%$. In the FE simulation, the target thickness reduction corresponded to the roll gap setting, and the resulting thicknesses might be different from the roll gap. Therefore, the thickness reductions and deformation parameters were calculated by the same method as in the experiment using the geometries before and after rolling simulation. In Fig. 12, the flange spread parameters $\ln \left(B_{1} / B_{0}\right)$ in the FE analysis (FEA) are plotted against the

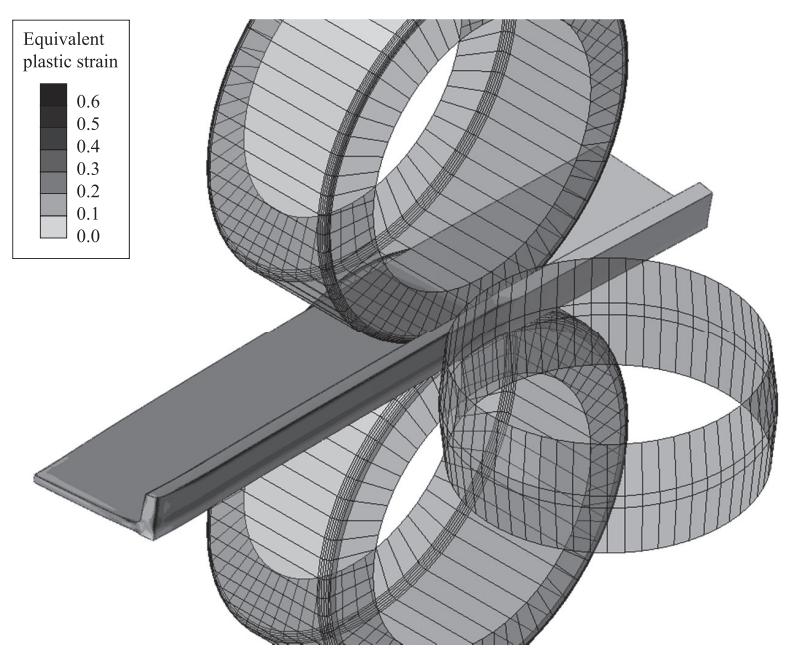

Fig. 11. Rolling deformation and equivalent plastic strain $\left(r_{w}=\right.$ $15 \%, r_{f}=18 \%$ ).

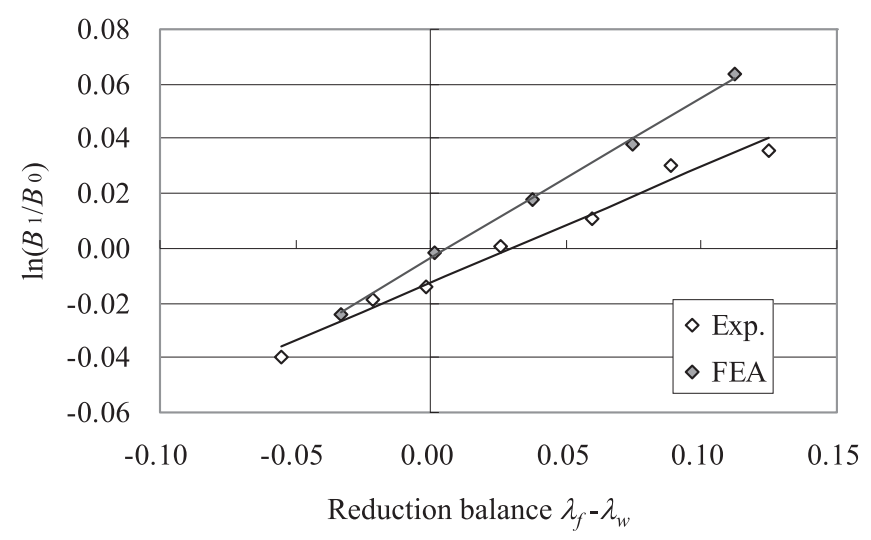

Fig. 12. Comparison of flange spread behavior between FE analysis and experiment. reduction balance with the experimental results (Exp). The FE results have a similar linear trend to the experimental results. However, the flange spread of the analysis at higher reduction balances is larger than that in the experiment, and this causes the inclination of the regression line to increase.

Figure 13 shows the spread parameter of web inner height $\ln \left(L H_{1} / L H_{0}\right)$ by FE analysis and the experiment. The results of the numerical simulation were in good agreement with the experimental results, including the unexpected trend of the positive inclination of the regression line.

To compare the results of $\Delta d$ and $\Delta h$, the experimental data were multiplied by 5 times for conversion to the actual dimensions. The relationship between the reduction balance and $\Delta d$ is shown in Fig. 14. The FE results are close to the experimental results, except in the high reduction balance region over 0.05 . The flange depth $\Delta d$ of FE analysis results did not change linearly in the high reduction balance region.

Figure 15 presents the results of bulge height $\Delta h$. Although the simulated results are slightly smaller than the experimental results, their trends are in good agreement.

As the result of FE analysis, the simulated deformation behaviors generally agreed with the trend of the experimental results except for the following differences: The inclination of the regression line for the flange spread parameter was rather lager, and the change of $\Delta d$ by FE analysis was not linear in the high reduction balance region. The causes of these differences are discussed in the next chapter.

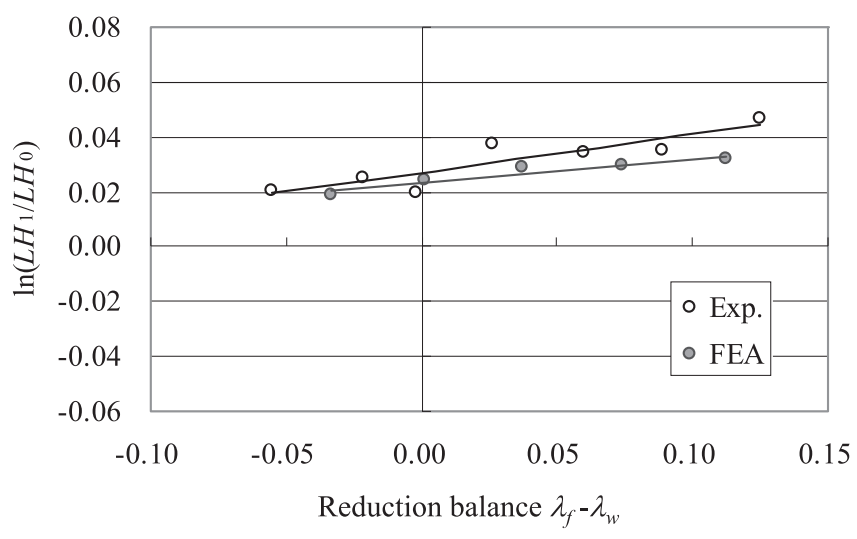

Fig. 13. Comparison of web spread behavior between FE analysis and experiment.

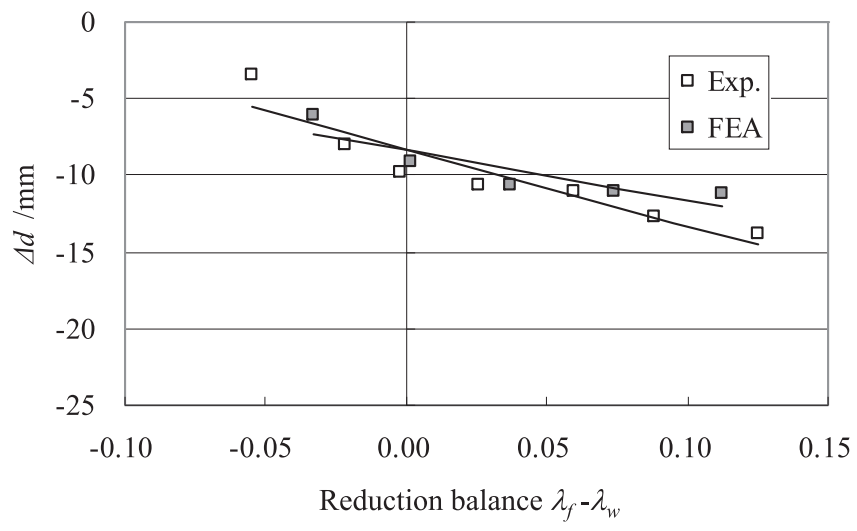

Fig. 14. Comparison of flange depth variation between FE analysis and experiment. 


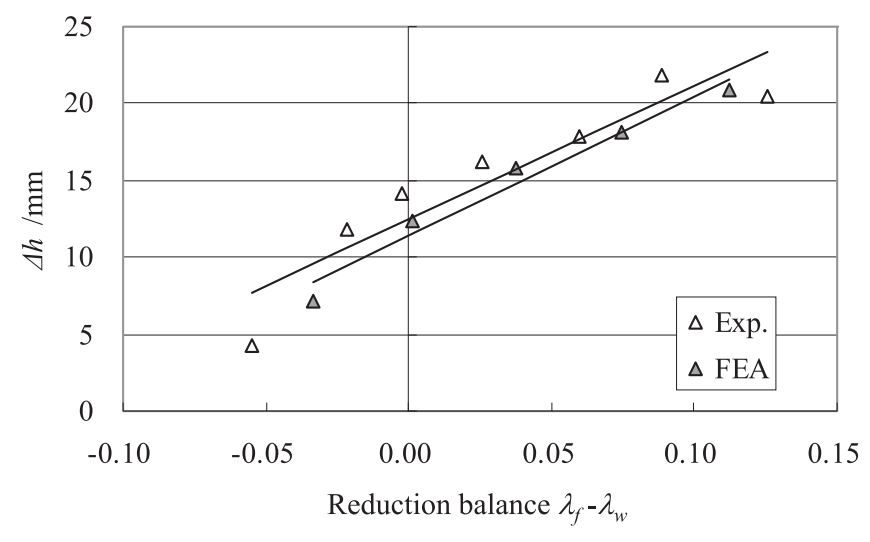

Fig. 15. Comparison of bulge height variation between FE analysis and experiment.

\section{Finite Element Simulation of Channel Universal Rolling}

It has been reported that the flange spread behavior of a T-bar having the half-section of an H-beam is similar to that of the original H-beam. ${ }^{3)}$ The same relationship was expected between an unequal-leg angle and a channel. Channel universal rolling is already used in some actual section rolling facilities. Therefore, if the affinity between unequal-leg angles and channels can be clarified, knowledge regarding channel rolling can be transferred to unequal-leg angle universal rolling.

As the unequal-leg angle has an L-section, it can be considered to correspond to one-half of the U-section of a channel. A FE simulation of channel universal rolling was performed to investigate the similarity in the rolling deformation behaviors of the two types of sections.

\subsection{Numerical Conditions of Channel Rolling Simula- tion}

The same FE code as that used in the simulation of unequal-leg angle rolling was also applied to the channel rolling simulation. As shown in Fig. 16, a half part of the channel (U-section) was modeled for the analysis, and the cross section had the same geometry as the unequal-leg angle (L-section) in Fig. 10. A perpendicular axis of symmetry was placed at the broken line in Fig. 16. Other conditions of the channel rolling simulation were determined so as to be same as those shown in Table 2 .

\subsection{Results of Channel Rolling Simulation and Discus- sions}

The sections of the unequal-leg angle and channel after rolling under the three $r_{f}$ conditions are presented in Fig. 17. As is evident in this figure, the vertical positions of the flanges of the two products were not similar.

Figure 18 shows the comparison of $\ln \left(B_{1} / B_{0}\right)$ between the two products. The flange spread of the L-section was always larger than that of the U-section.

Because of its symmetric section, the U-section has no spread of $L H$. On the other hand, the L-section shows unexpected spread of $L H$ as described in Chapters 2 and 3.

The change of flange depth $\Delta d$ in Fig. 19 shows an obvious difference. Although both products have some decrease

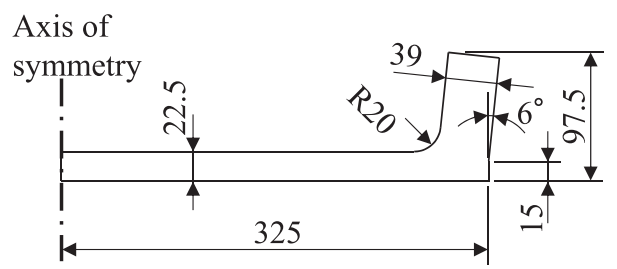

Fig. 16. Channel section before rolling.

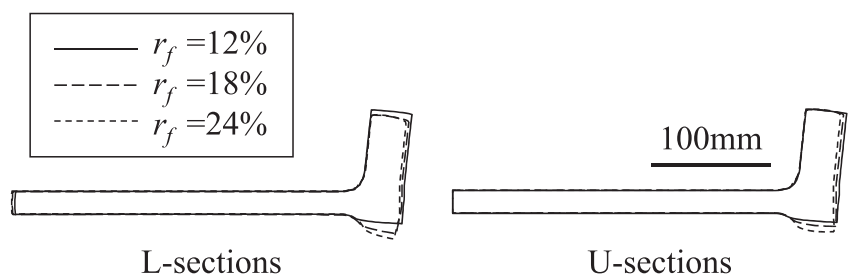

Fig. 17. Sections after rolling (FE analysis).

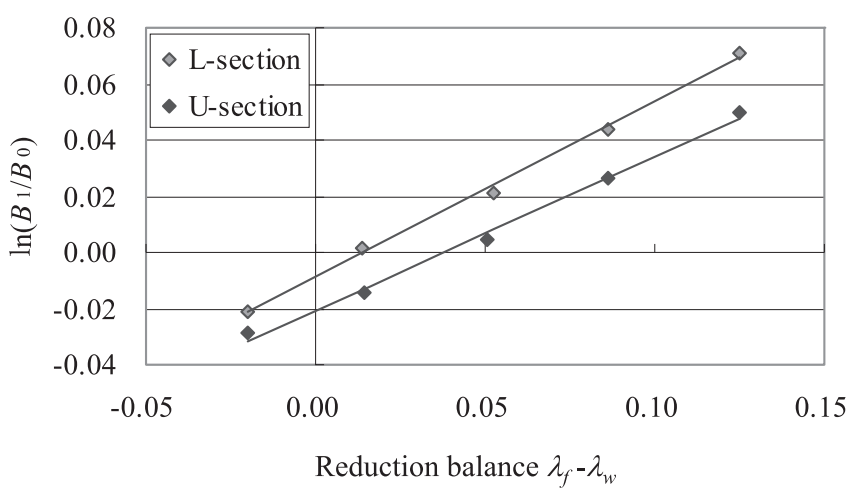

Fig. 18. Flange spread behavior of $L$ and U-sections.

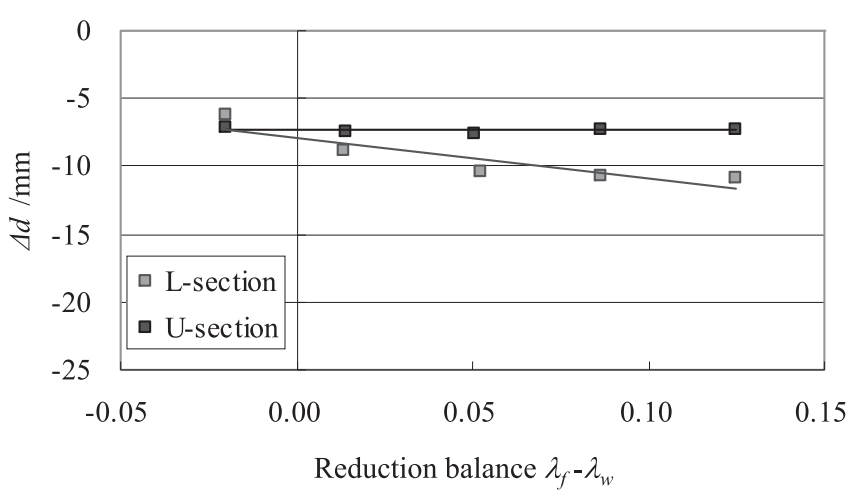

Fig. 19. Flange depth behavior of $\mathrm{L}$ and U-sections.

of flange depth, $\Delta d$ of the L-section decreases as the reduction balance increases, while that of the U-section remains almost constant.

The results of bulge height $\Delta h$ are shown in Fig. 20. At the reduction balance of -0.02 , the two products have similar $\Delta h$ values. However, the inclination of the regression line for the L-section was almost double of that of the Usection. As a conclusion, it was discovered that the two products have less similarity in their universal rolling deformation behaviors than in the case of H-beams and T-bars.

To clarify the cause of the difference between the L- and U-sections, the deformation history in the rolling simulation was examined in detail. Figure 21 shows the sections 


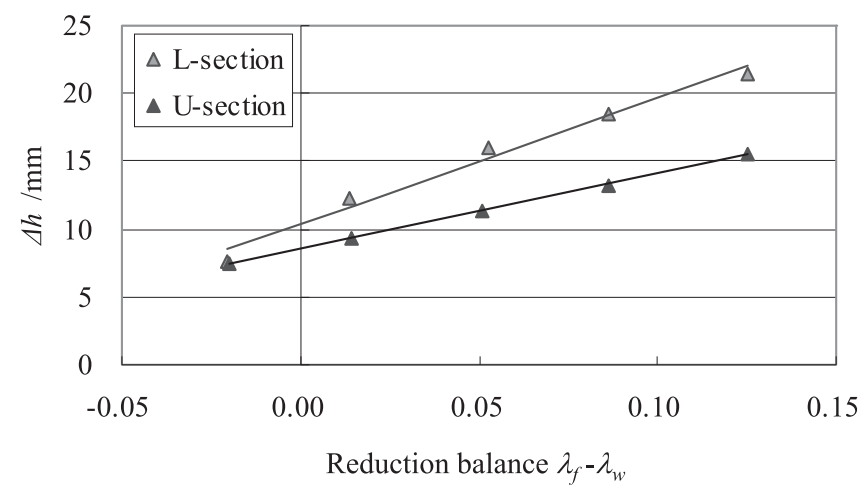

Fig. 20. Bulge height behavior of $\mathrm{L}$ and $\mathrm{U}$-sections.

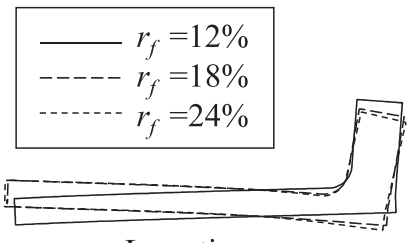

L-sections

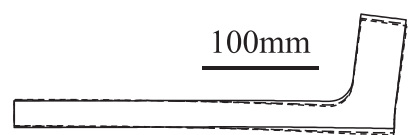

U-sections
Fig. 21. Sections at position $150 \mathrm{~mm}$ upstream from plane of roll axes.

$150 \mathrm{~mm}$ upstream from the plane of the roll axes. The outlines of the three $r_{f}$ conditions are illustrated for both the Land U-sections. Although the L-section has different rotation angles depending on $r_{f}$, the positions of the U-section are much more stable except for slight web bends. It is assumed that the symmetry of the U-section contributes to higher stability during rolling deformation, and this in turn contributes to smaller rolling deformation than in the Lsections.

The L-sections in Fig. 21 show that the web tip position shifts toward the left with the increases in the reduction balance due to stock rotation. From this, it is deduced that the unexpected spread of the web inner height observed in the experiment and FE analysis was caused by rotation at the entry side of the universal mill.

Therefore, the stability of the stock before rolling is essentially important for reducing undesirable large rolling deformation in unequal-leg universal rolling. For example, suitable positioning of the entrance guides makes it possible to decrease stock rotation before rolling. In the model rolling experiment in Chapter 2, downward elastic movement of the entrance table were observed. On the other hand, the entrance table in the FE simulation in Chapter 3 was assumed to be a fixed rigid surface set at $30 \mathrm{~mm}$ below the top of the lower horizontal roll. The differences of the table position and stiffness in the experiment and the FE simulation must be the reason for the mismatch of their $\Delta d$ tendencies. The cause of the different inclination of the regression lines in Fig. 12 is also thought to be related to the degree of stock rotation, but a further detailed investigation will be necessary in order to determine the actual cause.

The comparison of universal rolling deformation of Land U-sections clarified the fact that stock rotation before rolling is an important property in universal rolling of unequal-leg angles. Thus, improved stock stability at the entry side is indispensable for successful universal rolling of unequal-leg angles.

\section{Conclusions}

In this paper, deformation behavior in universal rolling of unequal-leg angles was investigated in detail by a laboratory model experiment and FE analysis.

The experimental results showed a flange spread behavior similar to that in universal rolling of H-beams, T-bars and channels. However, an unexpected increase of the web inner height was observed. Asymmetric flange deformation like that in universal rolling of channels was also observed in universal rolling of unequal-leg angles.

A three-dimensional non-steady-state FE simulation was carried out, and the tendencies of the simulated deformation were substantially similar to those in the experiment. However, two differences were noted: The inclinations of the regression lines for the flange spread parameter were larger, and the change of the flange depth against the reduction balance was not linear in the high reduction balance region.

Universal rolling deformation of a channel having the same half-section as the unequal-leg angle was simulated with the FE program. Stock rotation depending on the reduction balance was discovered in unequal-leg angle rolling from a comparison of the rolling deformation behaviors of the unequal-leg angle and channel. This clarified the fact that the tendencies of unequal-leg angle rolling deformation can be explained by stock rotation. The asymmetry of unequal-leg angle rolling was considered to be the reason for the unsteady stock rotation.

Prevention of stock rotation is an important factor for realizing universal rolling of asymmetric L-sections. Development of methods for stabilizing the stock position before rolling is the next step for establishing a universal rolling technology for unequal-leg angles.

\section{REFERENCES}

1) T. Misawa, K. Nakayama, K. Ototani and T. Makino: 2nd Int. Conf. Steel Rolling, Düsseldorfer Messegesellschaft $\mathrm{mbH}$, Düsseldorf, (1984), A10.

2) Y. Takashima and J. Yanagimoto: Steel Res. Int., 82 (2011), 1240.

3) Y. Takashima and T. Hiruta: ISIJ Int., 52 (2012), 1328.

4) Y. Takashima and T. Hiruta: ISIJ Int., 53 (2013), 690.

5) R. Young: Iron Steel Technol., 4 (2007), 41.

6) I. Kyoi, K. Nakajima, K. Isozumi, K. Kishikawa and K. Watanabe: Proc. Japanese S54 Spring Conf. Technol. Plast., Jpn. Soc. Technol. Plast., Tokyo, (1979), 473.

7) K. Nakajima, K. Watanabe, I. Kyoi, K. Isozumi and M. Kodama: Seitetsu Kenkyu, (1979), No. 299, 78.

8) I. Nakauchi, T. Hirasawa, T. Ide and Y. Suzuki: CAMP-ISIJ, 1 (1998), 505.

9) R. E. Beynon: Roll Design and Mill Layout, Association of Iron and Steel Engineers, Pittsburgh, (1956), 159.

10) E. E. Brayshaw: Rolls and Rolling, Blaw-Knox Company, PA, (1958), 260.

11) H. Tokita, S. Hamauzu, K. Watanabe and Y. Nishikawa: Tetsu-toHagané, 68 (1982), S1163.

12) Zosen-to-Kouzai, Kouzai-Kurabu, Tokyo, (1993), 51

13) K. Nakajima: Shape Rolling Technology of Steel, Chijin Shokan Co., Ltd., Tokyo, (1999), 213.

14) Y. Misaka and T. Yoshimoto: J. JSTP, 8 (1967), 414. 